\title{
Exploring a Hybrid Control Approach for enhanced User Experience of Interactive Lighting
}

\author{
Serge Offermans, Harm van Essen, Berry Eggen \\ Eindhoven University of Technology, Dept. of Industrial Design \\ Postbus 513, 5600MB, Eindhoven, the Netherlands \\ \{s.a.m.offermans, h.a.v.essen, j.h.eggen\}@tue.nl
}

\begin{abstract}
Modern lighting systems allow for light settings that are more in tune with users' activities, by going beyond mere functional illumination. These systems have a large amount of controllable parameters such as intensity and colour of individual light sources. Using an autonomous control system is therefore an attractive option, especially since such control systems may also lead to reduced energy consumption. From a user experience point of view however, there are certain drawbacks to this automation.
\end{abstract}

This paper proposes a hybrid approach towards lighting control to create a dynamic balance between user control and system automation. Such a hybrid system has the ability to autonomously set the lighting according to its knowledge about the current context, while offering users the possibility to manually adapt the light settings. These manual adaptations can in turn be used by the system to learn about user preferences in various situations, and thereby to improve its future lighting suggestions.

To explore and evaluate this approach, a smart lighting system was developed as an initial implementation, and installed in a real office environment. The system employs a machine learning algorithm to achieve intelligent behaviour and provides users with an interface to control the lights and give feedback to the system. In a six-week study, the user experience of this initial implementation is evaluated. The results provide an insight in design considerations when adopting this approach for the design of smart lighting control systems. The considerations regard the type of machine learning, the degrees of freedom offered to the user, the insight in the system's decision making process, and the user interface.

\section{Lighting Control, User Experience, Hybrid Control, User-System Interaction, Machine Learning}

\section{INTRODUCTION}

In modern offices, people are often confronted with a form of automatic control over their lighting. This trend is part of the ongoing developments in the area of smart buildings. Although definitions of smart buildings vary widely, one could say that they mostly aim for reduced energy consumption, increased cost effectiveness and employee comfort (Wong et al., 2005). Smart buildings employ lighting solutions that use sensor input (mostly occupancy and illuminance level sensing) to create desirable lighting conditions. In basic systems, the light on an office floor simply turns on when presence is detected, whilst the more advanced systems adapt the lighting level per desk/office area according to varying flux of incoming daylight, by controlling both artificial light and the window blinds. The state of the art systems go one step further and employ Artificial Intelligence principles to create a balance between comfort and energy efficiency (Dounis \& Caraiscos, 2009). Indeed automated systems using occupancy sensing save energy compared to normal wall switches, whilst adding light level sensing and dimming, based on norms for different types of tasks, saves additional energy (Jennings et al., 2000; Singhvi et al., 2005).

Despite these positive effects, several studies have also reported the negative consequences of such automated lighting. With automated systems, people often experience a loss of perceived control over their lighting. As a result, they perceive their lighting to be of lower quality and are less satisfied with the lighting (Veitch, 2001). In turn, this may lead to a lower level of comfort, and thus reduced job satisfaction and productivity (P. R. Boyce, 
2003). In line with these findings are the results of a study by Newsham et al. showing that offering control increases work satisfaction and even performance (G. Newsham et al., 2004). These authors do not consider this to be the effect of control per se, but actually of the resulting light setting. This implies that people have personal preferences in particular circumstances, and when enabled to change the lighting, people could improve their own working environment and their performance. Interestingly enough, it has also been shown that when given the freedom, people choose lower lighting levels than suggested by the standards which would result in a decrease of energy consumption (Moore et al., 2002; Guy Newsham et al., 2009), rather than the expected increase compared to an automated system.

Apart from the automation of adjustable task lighting, ongoing developments in modern lighting technologies create new opportunities that go well beyond the mere functional illumination of the office environment. LED lighting technologies offer possibilities to create atmospheres and personal lighting that is more in tune with a user's activities and mental state, creating benefits in terms of visibility as well as in terms of psychological, physiological and emotional effects (Knoop, 2006). From a technological viewpoint we have a high degree of control over this lighting as we are potentially able to adjust the intensity, colour, colour temperature, position, direction and possibly even more parameters. However, this increase in control freedom also increases complexity, which means that it remains unclear how to provide this level of control to people in a way that they can comprehend.

So far we have identified two issues for smart lighting in office spaces: 1) the seemingly contradicting desire for both automation and user control of task lighting and 2) the increased control complexity that results from the use of versatile lighting, for instance to create atmospheres. These two issues both arise from new technological opportunities and regard the balance between user control and automation. We acknowledge the potential of autonomous lighting control to support people in the creation of suitable lighting conditions. However, what is considered 'suitable' by the system, may be different from what is considered suitable by one user, which may again be different from another user.

We propose a hybrid control approach in which individual users and the system together come to an understanding of what is suitable lighting depending on the situation. Neither user nor system are fully in control, but rather the user influences the system's autonomous behaviour to varying extents. The primary aim is to enhance the user experience which in turn has an impact on the acceptance and adoption of the system, and may result in a positive influence on job satisfaction, performance and energy consumption.

In the initial exploration of this approach we adopt basic principles of machine learning to enable the system to make 'smart' decisions and user to influence the system. The core mechanisms for such a system are twofold. First, through machine learning, the system is enabled to learn about individual user preferences for various situations. Second, the users are offered control over the system allowing them to override system decisions, and moreover to 'teach' the system. There is a double gain here, as people are offered control when they would like to have it, whilst also getting more appropriate lighting from the autonomous system.

There are several others that propose the use of artificial intelligence in smart buildings, mostly to reduce energy consumption (Torunski et al., 2012). These systems use algorithms to predict the most energy efficient lighting based on previous measurements. What we aim to add to these approaches is the focus on user control, user experience, individual user preferences and effects of lighting beyond the visibility component.

In this paper we present an initial implementation of the proposed hybrid approach to explore its potential. The system that has been developed for this purpose uses a supervised learning approach with a rule based classification algorithm to determine the desired lighting output in various situations. The prediction of what is desirable lighting relies on a set of six input parameters (or features) that describe the current situation, such as the user's identity and the amount of natural light. Based on lighting choices made in the past in similar situations, the system chooses the most suitable lighting output out of eight lighting presets that are intended to support these situations. The system was installed in a real office environment and used in a six week field-evaluation. The goal of the implementation and the evaluation was to explore the potential of the hybrid approach, as well as to gain insight in the important aspects that influence the user experience of such systems.

In section two, we will introduce our experimental environment, the lighting design, the machine learning implementation and the user interface. In section three we present the field study in which the trained algorithm was deployed for six weeks in a living-lab context. Finally in section four we present the conclusions and reflect on the important aspects of the implementation that influence the user experience, which in turn can inform the design of future smart lighting systems. 

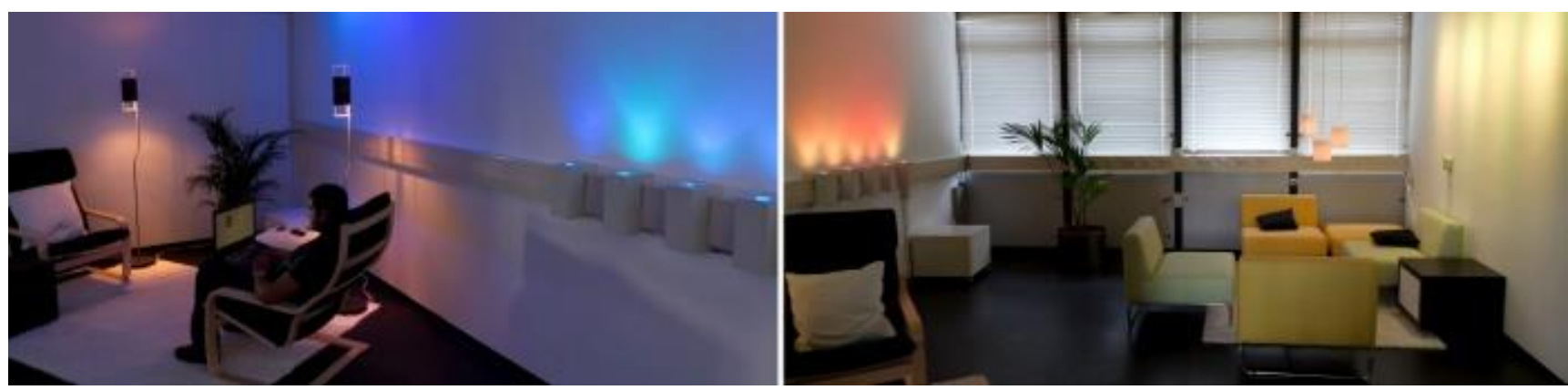

Figure 1. Living-lab area; (left) retreat area, (right) meeting area

\section{SYSTEM DESCRIPTION}

As an initial implementation of the proposed hybrid approach we have developed a system that consists of an environment with adaptable lighting

and various sensors, a smart-phone interface for users to control the lighting and a machine learning algorithm to predict and actuate the proper lighting in different conditions. In this section we will describe the system implementation. First, the environment and the available lighting are described, followed by an explanation of the machine learning algorithm and the user interface. Finally, we describe a typical usage scenario of the system.

\subsection{Living-lab area}

The system is implemented in a living-lab setup which functions as an area for informal meetings or personal retreat within our department (Offermans et al., 2012). This 'breakout' area is adjacent to an open plan office space which accommodates about 70 students and staff. The area allows people to leave their desks for a while, which gives them a change of scenery and allows them to work without being interrupted by e-mails or phone calls. The

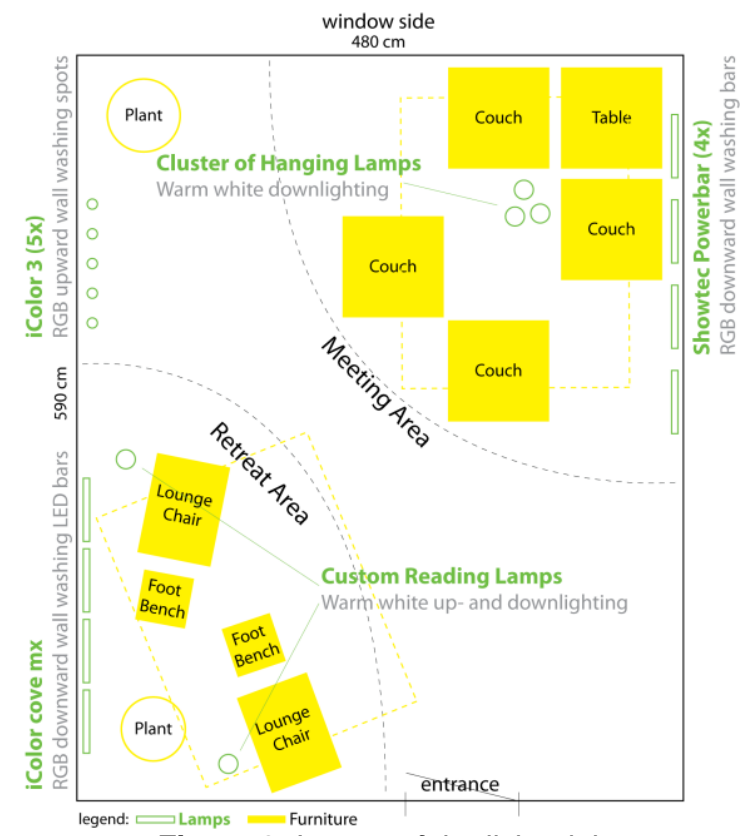

Figure 2. Layout of the living-lab area area is roughly divided in two areas; a retreat area with two lounge chairs and a meeting area with four couches (see Figures 1 and 2).

The area features a lighting system with coloured wall washing lights for atmospheric purposes and warm white down-lights for task lighting. All lights can be individually controlled in terms of intensity, and the wall washing lights can also be adjusted in colour. The system also has several sensors. Two Passive Infra Red (PIR) sensors monitor movement in the two separate areas, a sound pressure meter monitors general 'loudness' and a light level meter measures general light intensity in the room.

This particular type of area was chosen for its diverse usage compared to a desk work area, and therefore presents the system with a possibility to diversify lighting and distinguish between activities.

\subsection{Lighting design and presets}

The system in the area uses all available lamps to create a particular setting. As the initial implementation of machine learning relied on a number of lighting presets (rather than an infinite number of possible light settings based on continuous parameters), we defined eight output presets. We aimed to make these presets representative of a broader description of lighting in order to be able to generalize results for other environments. We therefore defined three lighting parameters along which the presets would be designed; brightness, warmth and dynamics. Together with a lighting designer, eight presets were created in the relative extremes of the parameter space, resulting in eight outputs (see Fig. 3).
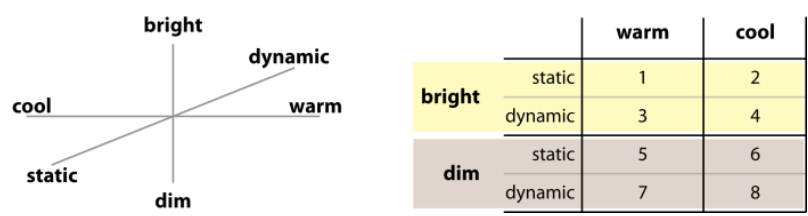

Figure 3. The lighting output parameters (left) and the resulting presets (right)

An impression of the resulting light presets can be found in Figure 4, and could be described as follows: 


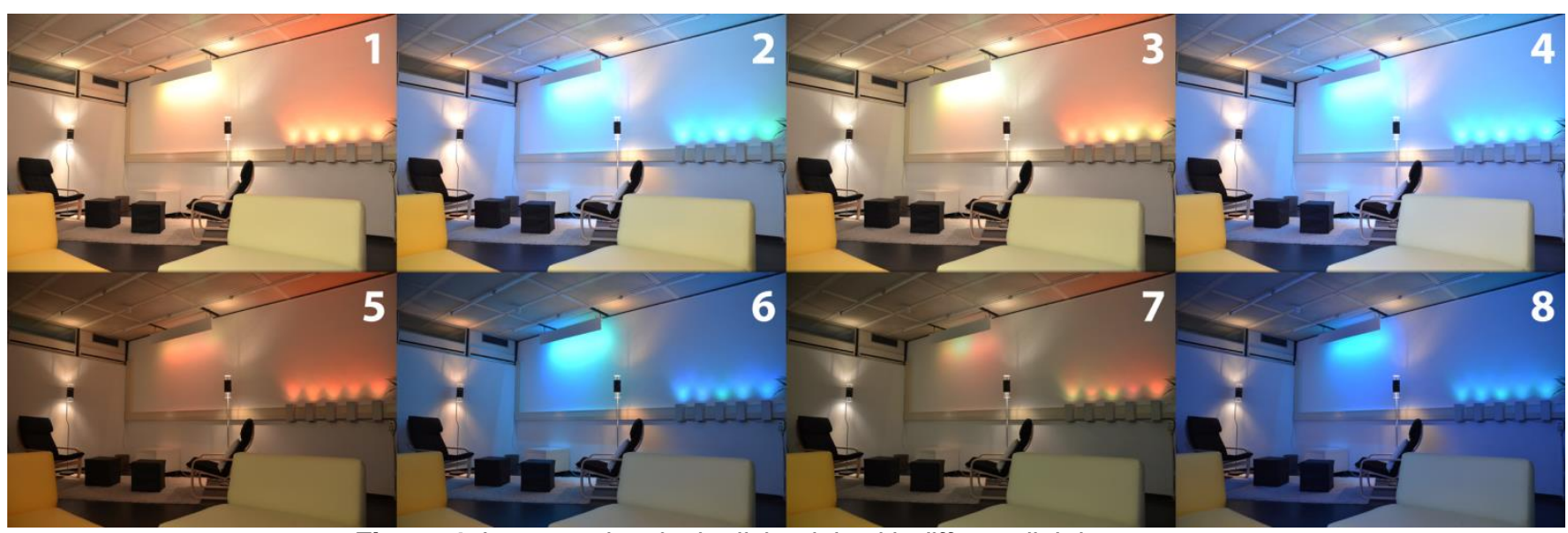

Figure 4. Images taken in the living-lab with different lighting presets

(i) Warm-Red/Orange, saturated colours, little colour variety, very slowly changing colours

(ii) Cool-Blue, semi-saturated, little colour variety, very slowly changing colours

(iii) Red-Yellow, saturated colours, more colour variety, colours changing at visible pace

(iv) Cyan-Purple, semi-saturated, more colour variety, colours changing at visible pace

(v-viii) Similar to i - iv, however dimmed.

To verify whether the light presets did indeed match the parametric descriptions (in terms of intensity, warmth and dynamics) from which they were created, a pre study was conducted in which

13 participants were shown four of the eight presets and asked to rate the lighting according to the three parameters (Dim - Bright, Warm - Cool, Dynamic - Static) on a 7-point scale. On average, participants scored the light settings as was expected from the intended design (i.e. the preset that was intended to be warm was scored as such). As can be seen in Figure 5, there were only two exceptions. First, Cool-Dim-Dynamic lighting was considered bright rather than dim. Second, WarmDim-Dynamic lighting was considered slightly static instead of dynamic. In general, the lighting was found to be more static than dynamic.

Regarding the representative value of the lighting designs for the output parameters, the warm and cool atmospheres clearly express this warmth parameter. Also for the bright-dim parameter, the overall difference was clearly recognized, although dim cool dynamic light was seen as bright. This may have been the result of the relatively large visible colour variety (lots of green) in this setting, which is also indicated by the strong dynamic score of this preset. For the dynamic-static parameter results were less clear, although it seems that static settings are indeed considered static. We can therefore state that the lighting designs were representative for their parametric description in terms of intensity and warmth, but not in terms of dynamics. Therefore the knowledge gained about preferences can be generalized in terms of intensity and warmth, and potentially transferred to other environments.

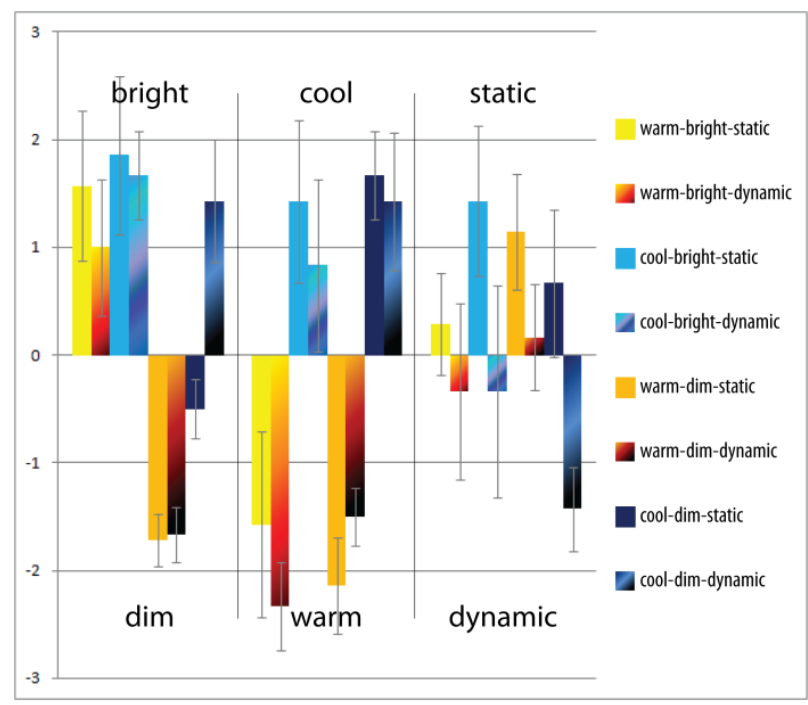

Figure 5. Overview of the average scores on the lighting design parameters

\subsection{Machine learning and lighting prediction}

To be able to predict suitable lighting based on the user's previous lighting choices, the system employs a machine learning algorithm. For the initial exploration a supervised learning approach was chosen as it is relatively simple to implement. The prediction system relies on several input parameters (or features) that are known to the system. Based on lighting choices that users made in the past in similar conditions, the system selects the most suitable lighting output. The input features used in this implementation were user identity, type of activity, area in which the user will sit, time of day, incoming daylight and movement in the other area. Most of these features are measured by the system, however the type of activity and the area in which the user intends to sit have to be explicitly provided by the user. For the area, the user can choose between the meeting- and retreat area (see 
Fig 1). For the type of activity the user can select one of four activity types that are a combination of creative or relax and individual or in a group (see Fig. 6).

\begin{tabular}{c|c|c} 
& creative & relax \\
\hline individual & sketching & reading \\
\hline group & $\begin{array}{c}\text { supervisor } \\
\text { meeting }\end{array}$ & $\begin{array}{c}\text { informal } \\
\text { meeting }\end{array}$
\end{tabular}

Figure 6. Four categories of activities with example activities that could fit these types

A rule based classification algorithm was then used to determine the desired lighting output. The details of the applied algorithm are reported in (Gopalakrishna et al., 2012).

To provide the system with initial knowledge about what is considered desirable lighting, the system was 'trained' by the participants in a training session. During this session, the same 13 participants were asked to imagine themselves being in a scenario (the scenarios are described at the end of this paragraph), and set the lighting as they would like to have it in this particular scenario. All relevant environment parameters that would be used as input for the learning algorithm were varied. Participants could try as many settings as they pleased until they were satisfied with the final result They would then be asked to motivate their choice. This sequence would be repeated four times with different scenarios.

Participants used a smart phone interface to set the lighting when they were in the room (the same as presented in fig. 7.3). Before starting, they received a basic instruction about the control interface which contained icons representing the eight presets.

Four short textual scenarios were used to describe the setting in which participants were asked to imagine themselves to be. The scenarios were described such that they were representative for the four types of activities that the system would distinguish (active/relax and individual/group, as described before, see Fig. 6). Scenarios were verbally presented to the different participants in varying orders.

(v) Active Individual: You have a rough ideadirection for your design project. You are going to make some idea sketches to explore the potential of this direction

(vi) Relax Individual: You have been working hard and you just want to take some time for yourself and browse through some webpages

(vii) Active Group: You are having a weekly meeting with your coach/student about the ideas in your/his/her project

(viii) Relax Group: Two of your friends in the department are coming over for a cup of coffee and a chat
The session provided data that consisted of 52 lighting-preset selections of 13 users in four different situations (four selections per participant, one for each scenario). These situations can be described by the parameters that were used by the system as input (user identity, activity type, the chosen area, time of day, amount of daylight and movement in the other area). The lighting preference in relation to the input parameters can be used by the machine learning algorithm to predict suitable lighting in various conditions. In an evaluation of the algorithm's performance, it appeared that it produced prediction efficiencies that were below expectations (Gopalakrishna et al., 2012) (efficiency is a measurement for the quality of the prediction). However, as the purpose of the evaluation in this paper regards the user experience of the system, rather than the lighting predictions, the relatively low efficiencies are not problematic.

\subsection{User Interface}

The user interface that was created for the system serves two purposes. Firstly, it allows people to indicate the type of activity they are going to perform and the area in which they will do that. This information is used by the system to predict the suitable lighting and activate it. Secondly, the interface allows users to explicitly change the lighting if they are not satisfied with the system's prediction, by choosing one of the eight available presets.

The user interface was implemented as an Android smart-phone application that was developed for the purpose. It was intended to be as basic as possible, as the interface itself was not the focus of this study, but rather the experience of shared control with the system. After starting the application, the initial screen allows the user to choose from four activity types as well as to choose between the two areas (see Fig. 7.1). After this choice has been made, the system activates the lighting based on the prediction algorithm, and the user can either close the application or indicate the wish to adapt the lighting (see Fig. 7.2). If the latter choice was made; the user can select one of the eight light presets that are represented by a set of icons (see Fig. 7.3). During the evaluation, participants used their own smart phones to control the area. This also provided the system with information about user-identity, which was crucial for the algorithm. 


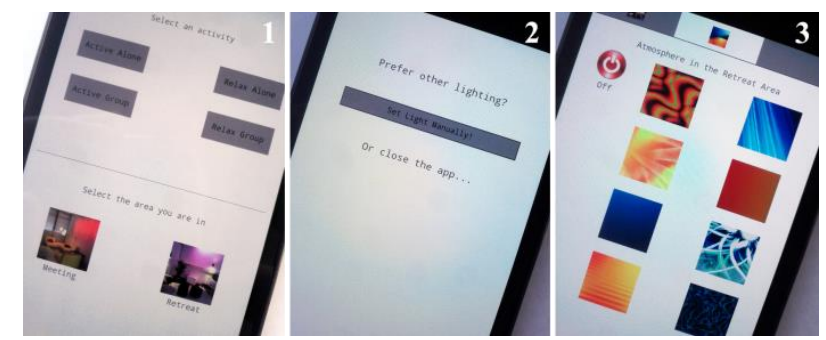

Figure 7. The user interface Android application. (1) Initial screen to choose activity type and usage area, (2) Screen displayed when activity and area were chosen, and when lighting is activated, (3) if the user wishes to adapt the lighting; he/she can manually select from the eight presets.

\subsection{Typical scenario of use}

To get an idea of how the system would normally be used, we describe a typical scenario of use.

When a user enters the area, he/she uses a smartphone application to select the type of activity that is intended (either active/relaxing and alone or in a group), as well as the area in which this will take place (meeting or retreat area). The system will then predict which of the eight lighting presets is most suitable and activate the lighting. If the user is satisfied he/she can close the application; if not, the user may choose to manually select the preferred setting. This information could again be used by the machine learning algorithm to improve future predictions, although this was not implemented in the initial system.

\section{FIELD EVALUATION}

To explore and evaluate the user experience of the initial system, we let the trained prediction algorithm run for a period of little over six weeks. During this period, the area could be used by the group of 13 participants that also trained the algorithm, as well as by other occupants of the adjacent open-plan office. All participants were either staff or students from our university who worked in close proximity to the living-lab, so they could use the area as part of their regular routines.

One of the main aims for this study was to gain insight in the usage and user experience of the system over a prolonged period of time. We were interested in the frequency of use, acceptance of the suggested lighting, the interface and the general concept of a learning system to provide suitable lighting.

\subsection{Study Setup}

During the six-week period, the participants could use the living-lab area how they pleased and as often as they pleased. When they would use the smart phone application in the area, the system would set the lighting based on the prediction algorithm. This algorithm would take into consideration all the input features described in section 2.3. Furthermore, participants would have the opportunity to manually adapt the lighting output through the smart phone interface. Due to the nature of the implemented learning algorithm, these adaptations could not be fed back into the learning algorithm during this study, so predictions were only based on the choices made during the training sessions.

During the usage period, all interactions with the system were logged, and the experimenters had informal conversations with the participants about their experiences with the system. After the usage period, participants were also asked to fill out a brief additional questionnaire. The questionnaire regarded the usage of the area and the control application; especially focussing on participants' motivation for use. The questionnaire was also intended to evaluate the experience of control and the system's smartness; more specifically it's lighting suggestions. We will now present the results and discuss them in section 4 .

\subsection{Quantitative Results}

In total, 79 interactions with the system were registered from 10 different users (an interaction was defined as usage by a single participant in which light changes were separated by less than 3 minutes). In 37 cases, people accepted the initial system suggestion (47\%); in 6 cases, people only requested a new prediction; in the remaining 36 $(46 \%)$ cases, people manually selected a new lighting condition. The new selection on average varied on 1 parameter (stdev $=0,6$ ) in comparison to the initial prediction. To manually find the lighting condition they preferred, people on average tried 4 $(3,83$; stdev $=2,7)$ different settings before they were satisfied. When people were not satisfied with the prediction, the duration of the interaction that followed to find the desired preference was 1 minute 8 seconds on average (stdev $=1 \mathrm{~min} .4$ sec.).

The interactions took place in 53 sessions (a session was defined as usage by a single participant with interactions that are separated by less than 60 minutes). In 17 cases (32\%), people used the application more than once during a session, with an average of 2,58 interactions per session (stdev $=0,91$ ). These interactions were separated by a time span of on average 17.45 minutes (stdev = 9 min. $44 \mathrm{sec}$.).

\subsection{Qualitative Results}

After the study period, an additional qualitative questionnaire was sent to the participants. All respondents $(n=5)$ indicated to have used the area between 4 and 10 times in the period of 6 weeks; 
which is consistent with the logged data. We will first discuss the results of these questionnaires and afterwards discuss the results of the informal conversations.

All participants used the mobile phone application, as it was the only means to control the light. Whilst one person liked the personal aspect of the mobile phone, another indicated that taking the mobile phone out of his pocket broke the flow of entering the space and getting to work.

The lighting suggestions provided by the system were considered appropriate, but the participants indicated they were unable to get the reasoning of the system and felt as if most of the lighting conditions would have been suitable. In turn, this lack of understanding lead to a form of indifference towards the smartness of the system, and participants simply accepted what was suggested or changed it to a setting of their liking.

All participants indicated that the control provided by the smart-phone application was good, but relatively limited (i.e. only eight presets) considering the freedom that the lights could technically provide.

Regarding the smartness of the system, people indicate that they thought it is conceptually interesting and relevant, but that they did not experience the system's smartness during this period. The main motivations that were mentioned for this, were the low frequency of use, the lack of insight in the system's decision making process and the system's inability to keep learning during the evaluation period (predictions were only based on the lighting choices made in the training session). Also one participant questioned the smartness of the system as there are probably too many relevant parameters for the system to take into consideration for predictions. Another participant doubted whether his own lighting choices were consistent enough for the system to learn. On the other hand, participants generally considered the lighting predictions to be suitable. Finally, two people suggested better integration with daylight control; which can currently only be obtained by manually closing or opening the venetian blinds.

Many of the results mentioned in this section, also came forward during the informal conversations throughout the evaluation period. Participants addressed the fact that the system did not express its 'smartness' and especially learning ability. Some felt that they would be more inclined to interact with the system if the effects of their interaction efforts would be more visible, and if they would have a better insight in the system's decision making process. Also the issues of relatively limited light setting options and the smart-phone based interface were mentioned.

\section{DISCUSSION AND CONCLUSIONS}

In this paper we have proposed a hybrid approach towards lighting control, and presented and evaluated an initial implementation of a system that uses this approach to create a balance between user and system control. Our aim was to explore the potential of this approach and to gain insight in the important aspects of such systems, especially in terms of user experience.

The results of the study show that participants adapted the automated lighting in approximately half of the cases which indicates that both system automation and manual control were appreciated. This may partially be attributed to an unsatisfactory lighting prediction, however judging from the qualitative results, this may also be a result of the general desire to have control over the lighting environment. This supports the case for the hybrid control approach, and indicates the potential for the use of machine learning in lighting control, which is in line with the findings presented by Torunski et al. (Torunski et al., 2012). Moreover it affirms the importance of an active role for the user in lighting control which we strongly advocate. The results also indicate that people sometimes (in about 1/3rd of the sessions) desire a variation in lighting during a session, and used the system to acquire this change. For a future implementation, we aim to explore the use of changes in lighting not only at the beginning, but also during the session.

From the design and evaluation of the implementation, several considerations for the design of hybrid control systems came forward which may inform the design of future lighting control systems. The considerations regard the freedom of control, the type of learning, the insight in the system's decisions and the user interface.

First, the exploration sheds a light on the relevance of the control freedom that is offered to the user. In the current implementation, the eight lighting presets provided relatively little freedom in control. This meant that people were forced to use a predefined setting that most likely differs (at least to some extent) from their personal preference. Furthermore as the settings were the result of a parametric design exercise, they often appeared rather similar to other settings (e.g. there were only two colour schemes). Apart from the limitations this poses for the users, it also limits the learning abilities for the system, as the feedback provided by the users most likely does not match their more detailed preferences. The development of a system that uses continuous parameters instead is part of future work. Freely adjustable brightness, temperature and dynamics will be made available 
to the users, as well as being used by the system for lighting prediction. We believe that one should aim to provide the available degrees of freedom to the user as long as this can be done in a comprehensible manner. It is likely this will improve both the system's learning capabilities as well as the user's feeling of control.

Second, for this exploration, the choice was made to use an implementation of supervised learning which meant that the system was not able to do 'online learning' (i.e. immediately use the manual adaptations made by the users to learn and improve future predictions). As users were aware of this, they knew that adapting the lighting would only contribute to their immediate experience and have no long term effect on the system's behaviour. In turn, this reduced some of the users' motivation to adapt the lighting. This aspect of the initial implementation contrasts our proposition in which machine learning is used as a hybrid mechanism that would allow user and system to actively and continuously collaborate on the light settings. To achieve this it is important that systems have a continuous learning process as opposed to a dedicated training phase. In general, it appeared that the specific machine learning approach used in the initial exploration; supervised learning was relatively limited and not the most suitable approach for the context. It was however chosen as an initial implementation to explore the opportunities and important aspects of machine learning for this context, and did indeed provide us with numerous insights. In future work we aim to use a reinforcement learning approach which will allow us to deal with the limitations mentioned above.

Third, the importance of the insight that users have in the system's learning and decision making process became apparent. In the initial implementation this insight was not explicitly provided, which hindered the collaboration between user and system. People did not understand how and why a certain light setting was chosen. This lack of understanding in some cases lead to indifference towards the system, rather than the urge to support its learning process. We believe it is important to provide the user with insight in the systems decision, which is in line with the concept of intelligibility as proposed by Belotti and Edwards (Bellotti \& Edwards, 2001).

Finally, if we wish to elicit interaction with the system to provide user-control and to feed the learning process, this interaction should match the flow of the activity that is going on or initiated. In our initial implementation, the mobile phone application was the only available interaction mechanism which was in some cases considered disruptive as control was not readily available. Besides manually changing the lighting, the user also had to use his/her smart phone to explicitly provide input to the prediction algorithm about the intended activity. Both this input for the prediction, as well as manually adapting the lighting would better be done through an interface that is more easily accessible, and for instance physically present in the area. This could further stimulate the dialogue between user and system and contribute to better balance between user and system control.

Concluding, we have explored a hybrid approach for the control of modern lighting systems, through an initial implementation and evaluation, aiming to achieve a balance between user and system control. An evaluation of the initial implementation provided several considerations that can inform the design of future lighting control systems and contribute to an enhanced user experience.

\section{ACKNOWLEDGEMENTS}

This research is part of the Smart Context-aware Services (SmaCS) project which is funded by the Point-One program. We would like to thank all participants in the studies for their effort and feedback. Furthermore we would like to thank Aravind Kota Gopalakrishna of the Eindhoven University, department of Computer Science for the implementation of the machine learning algorithm.

\section{REFERENCES}

Bellotti, V., Edwards, K. (2001). Intelligibility and Accountability: Human Considerations in Context-Aware Systems. Human-Computer Interaction, Vol 16(2-4), pp. 193-212.

Dounis, A. I., Caraiscos, C. (2009). Advanced control systems engineering for energy and comfort management in a building environment A review. Renewable and Sustainable Energy Reviews, Vol 13(6-7), pp. 1246-1261.

Gopalakrishna, A. K., Ozcelebi, T., Liotta, A., Lukkien, J. J. (2012). Exploiting machine learning for intelligent room lighting applications. In proceedings of IEEE $6^{\text {th }}$ International Conference Intelligent Systems, IS'12, Sofia, Bulgaria, 6-8 September 2012, pp. 406-411.

Jennings, J. D., Rubinstein, F. M., DiBartolomeo, D., Blanc, S. L. (2000). Comparison of control options in private offices in an advanced lighting controls testbed. Journal of the Illuminating Engineering Society, Vol 29, pp. 39-60.

Knoop, M. (2006). Dynamic lighting for well-being in work places: Addressing the visual, emotional and biological aspects of lighting design. In proceedings of $15^{\text {th }}$ International Symposium on Lighting Engineering, Bled, Slovenia. pp. 63-74 
Moore, T., Carter, D., Slater, A. (2002). User attitudes toward occupant controlled office lighting. Lighting Research and Technology, Vol 34(3), pp. 207-219.

Newsham, G., Veitch, J., Arsenault, C., Duval, C. (2004). Effect of dimming control on office worker satisfaction and performance. In proceedings of annual Illuminating Engineering Society of North America Conference, Tampa, Florida, pp. 19-41.

Newsham, G, Mancini, S., Veitch, J., Marchand, R., Lei, W., Charles, K., Arsenault, C. (2009). Control strategies for lighting and ventilation in offices: effects on energy and occupants. Intelligent Buildings International, Vol 1(2), p.p. 101-121.

Offermans, S., Kota Gopalakrishna, A., van Essen, H., Ozcelebi, T. (2012). Breakout 404 : A Smart Space Implementation for Lighting Services in the Office Domain. In proceedings of $9^{\text {th }}$ International Conference on Networked Sensing Systems, Antwerp, Belgium, 11-14 June 2012, pp. $1-4$
Boyce, P. R., Veitch, J. A., Newsham, G. R., Myer, M., Hunter, C. M. (2003). Lighting Quality and Office Work: A Field Simulation Study. doi:irc_id:15879

Singhvi, V., Krause, A., Guestrin, C., Garrett,Jr., J. H., Matthews, H. S. (2005). Intelligent light control using sensor networks. In proceedings of $3^{\text {rd }}$ international conference on Embedded networked sensor systems, New York, USA, pp. 218-229.

Torunski, E., Othman, R., Orozco, M., Saddik, A. E. (2012). A Review of Smart Environments for Energy Savings. Procedia Computer Science, Vol 10, pp. 205-214.

Veitch, J. A. (2001). Psychological processes influencing lighting quality. Journal of the Illuminating Engineering Society, Vol 30(1), pp. 124-140.

Wong, J. K. W., Li, H., Wang, S. W. (2005). Intelligent building research: a review. Automation in Construction, Vol 14(1), pp. 143159. 